\title{
Thermally Active TRPV1 Tonically Drives Central Spontaneous Glutamate Release
}

\author{
Kiyomitsu Shoudai, James H. Peters, Stuart J. McDougall, Jessica A. Fawley, and Michael C. Andresen \\ Department of Physiology and Pharmacology, Oregon Health and Science University, Portland, Oregon 97239
}

Central synapses spontaneously release neurotransmitter at low rates. In the brainstem, cranial visceral afferent terminals in caudal solitary tract nucleus (NTS) display pronounced, activity-dependent, asynchronous release of glutamate and this extra release depends on TRPV1 receptors (TRPV1+). Asynchronous release is absent for afferents lacking TRPV1 (TRPV1-) and resting EPSC frequency was greater in TRPV1 + . Here, we studied this basal activity difference by assessing thermal sensitivity of spontaneous and miniature synaptic events in TRPV1 + and TRPV1 - second-order NTS neurons. The spontaneous EPSC rate decreased when temperature was decreased, increased steeply between 30 and $42^{\circ} \mathrm{C}$ only in TRPV1 + neurons, and was calcium-dependent. TRPV1-specific antagonist SB366791, but not TTX, strongly attenuated thermal responses. Temperature changes failed to alter EPSC frequency in TRPV1- neurons. EPSC amplitudes and decay kinetics changed little with temperature. IPSCs in these second-order NTS neurons were unaltered by temperature. Such results suggest that activated, presynaptic TRPV1+ receptors trigger continuous resting release of glutamate vesicles at physiological temperatures only in capsaicin-responsive terminals. In mechanically isolated individual neurons harvested from medial NTS, increases in temperature increased the rate of glutamate release only in TRPV1+ neurons, whereas IPSC rates were unaffected. Cadmium failed to block thermal increases in glutamate release, suggesting that calcium entry through TRPV1 channels may trigger glutamate release independently of voltage-activated calcium channels. Together, our findings indicate a new form of afferent signaling in which TRPV1 channels within central terminals of peripheral afferents tonically generate glutamate release in NTS at $37^{\circ} \mathrm{C}$ in the absence of afferent action potentials.

\section{Introduction}

Quiescent central synaptic terminals often spontaneously release neurotransmitter at low rates and these events are thought to reflect the stochastic release of primed, docked synaptic vesicles. Rates of spontaneous release at rest are generally a thousand-fold lower than quantal release rates evoked by depolarization and it remains uncertain whether these two types of release are derived from a common or separate pool of vesicles (Wasser and Kavalali, 2009). TRPV1 is an ion channel activated in somatosensory primary afferent neurons during noxious heat and peripheral tissue damage (Lumpkin and Caterina, 2007). TRPV1 is also expressed at most presynaptic primary afferent terminals within the brainstem at the solitary tract nucleus (NTS) (Patterson et al., 2003; Sun et al., 2009) - a location unlikely to meet harsh TRPV1 gating requirements, including thermal thresholds of $\sim 43^{\circ} \mathrm{C}$ (Julius and Basbaum, 2001). Recently, we found that TRPV1 regulates

\footnotetext{
Received May 19, 2010; revised Sept. 4, 2010; accepted Sept. 9, 2010.

This work was supported by National Institutes of Health (NIH) Grants HL-41119 and HL-88894.

The content is solely the responsibility of the authors and does not necessarily represent the official views of the National Heart, Lung and Blood Institute or the NIH.

Correspondence should be addressed to Dr. Michael C. Andresen, Department of Physiology and Pharmacology, Oregon Health and Science University, Portland, Oregon 97239-3098. E-mail: andresen.ohsu@gmail.com.

K. Shoudai's present address: Research Division for Life Sciences, Kumamoto Health Science University, Kumamoto 860-5598, Japan.

J. H. Peter's present address: Department of Veterinary and Comparative Anatomy, Pharmacology, and Physiology, Washington State University, Pullman, WA 99164.

DOI:10.1523/JNEUROSCI.2557-10.2010

Copyright $\odot 2010$ the authors $\quad 0270-6474 / 10 / 3014470-06 \$ 15.00 / 0$
}

activity-dependent asynchronous release from a distinct pool of solitary tract (ST) afferent glutamate vesicles and the basal, prestimulation release rates from TRPV1+ were greater $(\mathrm{Pe}-$ ters et al., 2010). This TRPV1-sensitive asynchronous pool of vesicles could be specifically isolated pharmacologically or by decreasing temperature and was independent of the synchronous glutamate release responsible for ST-evoked EPSCs. The presence of a unique pool of TRPV1-dependent glutamate vesicles raises fundamental questions about the nature of the coupling between TRPV1, glutamate release, and presynaptic activity.

To investigate this unique vesicle population, we studied the mechanisms governing the unsynchronized, or "autonomous," release of glutamate in second-order NTS neurons in relation to TRPV1 expression and temperature. We assessed the roles of action potentials, local circuits, and calcium entry in the genesis of unsynchronized synaptic activity. Cooling and TRPV1 antagonism suppressed spontaneous EPSCs, suggesting that TRPV1 actively accelerates tonic release of glutamate from TPRV1+ afferent terminals. Glutamate release onto TRPV1- neurons or GABAergic transmission was thermally insensitive. Block of afferent terminal depolarization with TTX or voltage-activated calcium entry with cadmium did not prevent thermally evoked, calcium-dependent release of glutamate so that the calcium entry for vesicle fusion and release likely occurred through TRPV1 channels. Thus, since the majority of cranial visceral afferents are unmyelinated (Andresen et al., 2004), the presence of active TRPV1 in most cranial afferent terminals provides a new form of 
A

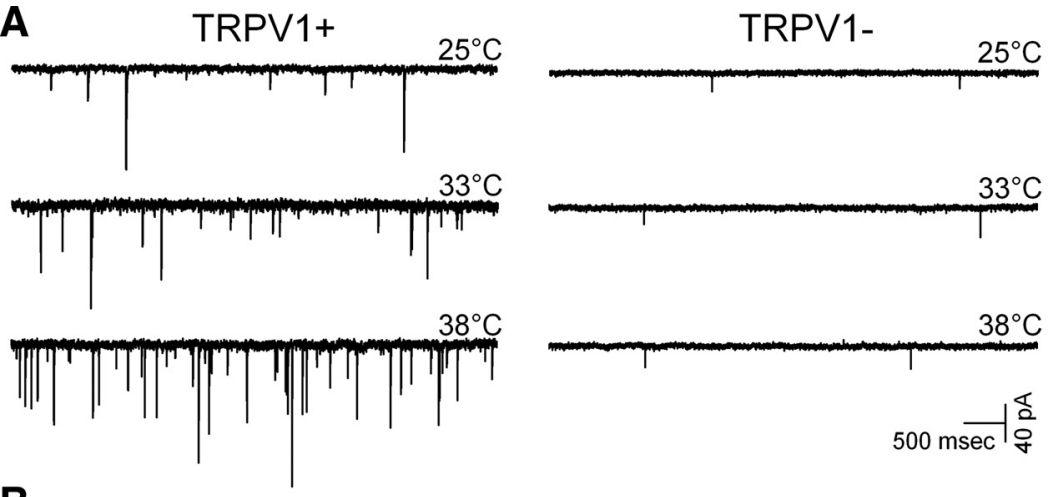

B
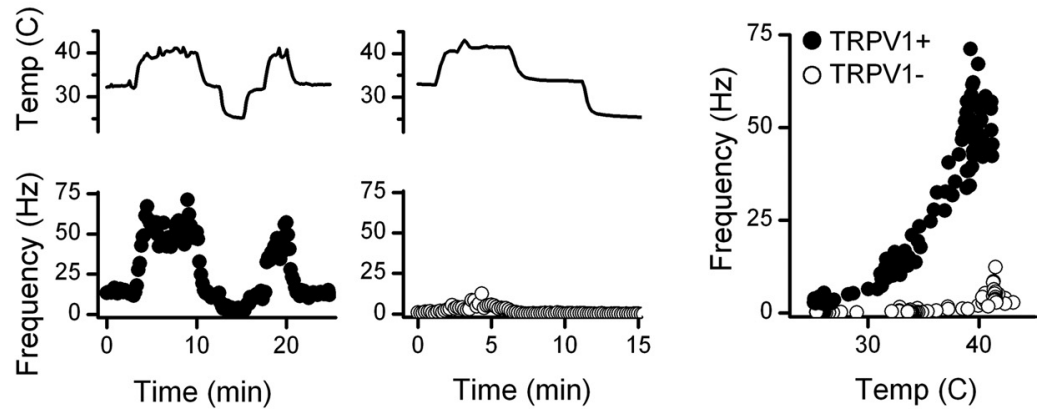

C
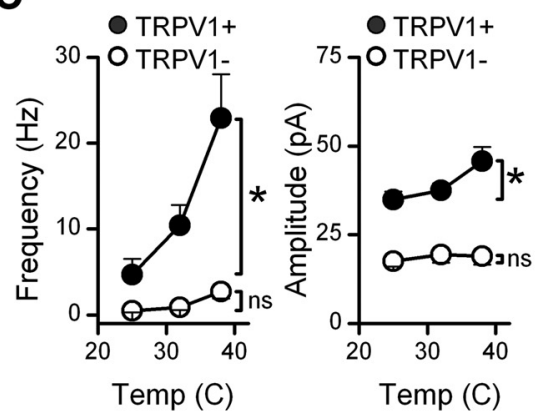

D

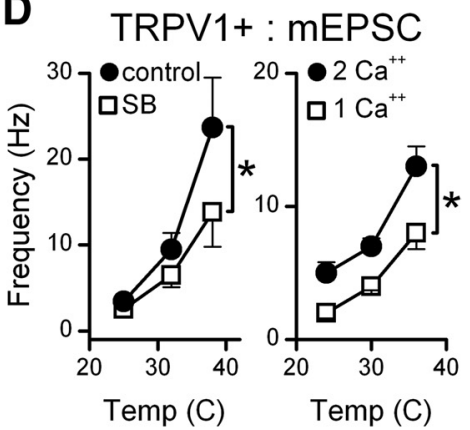

E
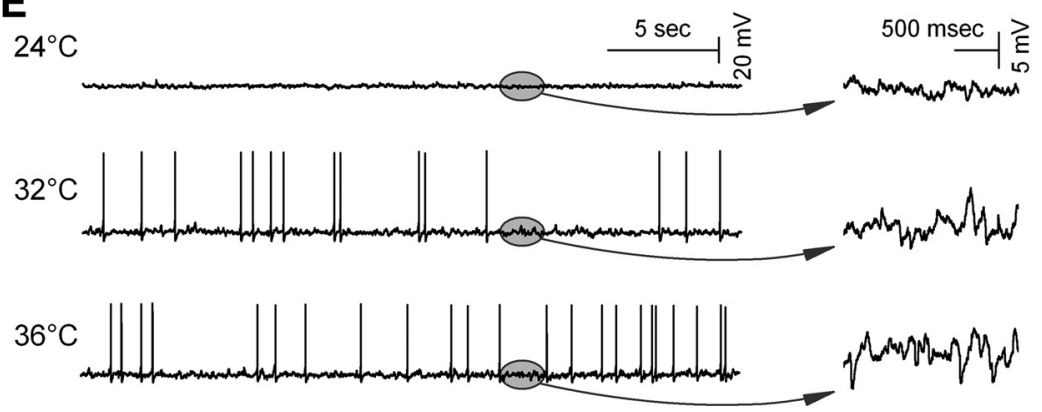

Figure 1. Temperature strongly augmented spontaneous glutamate release in TRPV1+ compared with TRPV1 - secondorder NTS neurons. $\boldsymbol{A}$, Original traces of sEPSCs from representative neurons (left, TRPV1+; right, TRPV1-) show that TRPV1+ neurons typically had higher spontaneous EPSC rates than TRPV1 - neurons at each temperature. $\boldsymbol{B}$, Temperature (upper trace) rapidly and reversibly changed spontaneous EPSC rates in a TRPV1 + (filled circles), but not in a TPRV1 - (unfilled circles), neuron. Frequency points are counts $>10$ s bins, expressed as average frequency. Note that $\boldsymbol{A}$ and $\boldsymbol{B}$ are from same neurons. $\boldsymbol{C}$, Aggregate summary for temperature responses of seven TRPV1 + (filled circles) and six TRPV1 - (unfilled circles) neurons, displayed as means \pm SEM. On average (two-way, repeated-measures ANOVA), temperature increased frequency in TRPV1 $+(p<0.001)$ but not in TRPV1 $-(p>0.2)$. sEPSC amplitudes increased slightly in TRPV1 $+(p<0.001)$. Decay time constants of sEPSCS (single exponentials, data not shown) declined similarly $(p=0.15)$ in both TRPV1 + and TRPV1 - , decreasing from 3.12 to $1.78 \pm 0.09$ ms from 25 to $38^{\circ} \mathrm{C}$. $\boldsymbol{D}$, In the absence of action potentials $(1 \mu \mathrm{M}$ TTX), mEPSC frequency increased with temperature. The TRPV1-selective antagonist SB366791 (10 $\mu \mathrm{m}, n=5$; left, unfilled squares) significantly decreased mEPSC activity at temperatures $>33^{\circ} \mathrm{C}(p<0.001$, two-way repeated-measures ANOVA). In separate TRPV1+ neurons ( $n=7$; right) temperature sensitivity of mEPSC frequency was reduced ( $p<0.001$, two-way repeated-measures ANOVA) by reducing bath $\mathrm{Ca}^{2+}$ (right) from control of $2 \mathrm{~mm}$ (filled circles) to $1 \mathrm{~mm}$ (unfilled squares) Average values within neurons were binned in $3^{\circ} \mathrm{C}$ intervals and represented by their midrange. Asterisks mark significant differences. $\boldsymbol{E}$, In a representative current-clamp example of five phenotype-specific control over synaptic signaling that generates a tonic stream of glutamate release even in the absence of peripheral afferent activation.

\section{Materials and Methods}

Brainstem slices. Horizontal brainstem slices $(250 \mu \mathrm{m})$ were prepared from adult male Sprague Dawley rats $(>160 \mathrm{~g})$ under isoflurane anesthesia, as previously described, using cooled artificial CSF (aCSF) containing the following (in mM): $125 \mathrm{NaCl}, 3 \mathrm{KCl}, 1.2 \mathrm{KH}_{2} \mathrm{PO}_{4}$, $1.2 \mathrm{MgSO}_{4}, 25 \mathrm{NaHCO}_{3}, 10$ dextrose, and 2 $\mathrm{CaCl}_{2}$, bubbled with $95 \% \mathrm{O}_{2}-5 \% \mathrm{CO}_{2}$ at 300 mOsm (Doyle and Andresen, 2001; Peters et al., 2008). All animal procedures were approved by the Institutional Animal Care and Use Committee and conformed to the guidelines of the National Institutes of Health publication "Guide for the Care and Use of Laboratory Animals.”

Slice recordings. NTS slices were perfused with aCSF at $34-35^{\circ} \mathrm{C}$, with temperature controlled using an in-line system (TC2BIP with HPRE2; Cell MicroControls). Bath temperature was continuously measured immediately downstream to the slice. Details for visualized recordings were as previously described (Peters et al., 2010). Recording electrodes (2.1-3.2 M $\Omega$ ) were filled generally with a low $\mathrm{Cl}^{-}\left(10 \mathrm{~mm}, \mathrm{E}_{\mathrm{Cl}}=-69 \mathrm{mV}\right)$, intracellular solution which contained the following (in $\mathrm{mm}$ ): $6 \mathrm{NaCl}, 4 \mathrm{NaOH}, 130 \mathrm{~K}$-gluconate, 11 EGTA, $1 \mathrm{CaCl}_{2}, 1 \mathrm{MgCl}_{2}, 10$ HEPES, $2 \mathrm{Na}_{2} \mathrm{ATP}$, and $0.2 \mathrm{Na}_{2} \mathrm{GTP}$. The intracellular solution was pH 7.3 and 296 mOsm. Neurons were voltage clamped at $\mathrm{V}_{\mathrm{H}}=-60 \mathrm{mV}$ with a MultiClamp 700B amplifier (Molecular Devices) in open, whole-cell configuration. Liquid junction potentials were not corrected. Signals were filtered at $10 \mathrm{kHz}$ and sampled at $30 \mathrm{kHz}$ using pClamp (version 9.4; Molecular Devices). In experiments targeting GABA-mediated IPSCs, glutamate receptor antagonists, NBQX $(20 \mu \mathrm{M}$, 2,3-dihydroxy-6-nitro-7-sulfamoyl-benzo[f] quinoxaline-2,3-dione), and AP-5 (D-2amino-5-phosphonopentanoate; $50 \mu \mathrm{M}$ ) were added and pipette $\mathrm{Cl}^{-}$content of the intracellular solution was adjusted to $54 \mathrm{~mm}$ and IPSCs were directed inward $\left(\mathrm{E}_{\mathrm{Cl}}=-22 \mathrm{mV}\right)$. Distal ST shocks (200 $\mu \mathrm{m}$ concentric electrode; Frederick Haer) identified second-order neurons by analysis of latency SD (jitter) of $<200 \mu \mathrm{s}$ (Doyle and Andresen, 2001) with ST shocks delivered every $6 \mathrm{~s}$ (duration $100 \mu \mathrm{s}$; Master-8; AMPI). At the end of each recording, capsaicin (100 nM) determined vanilloid sensitivity-TRPV1+ or TRPV1-.

Isolated neurons. Neurons were mechanically dissociated from horizontal slices (Jin et al., 2009). Standard external solution contained the following (in $\mathrm{mM}$ ): $150 \mathrm{NaCl}, 5 \mathrm{KCl}$, $1 \mathrm{MgCl}_{2}, 2 \mathrm{CaCl}_{2}, 10$ HEPES, and 10 glucose

$\leftarrow$

TRPV1+ neurons, synaptic potential rates increased substantially with temperature (expanded plots in ovals, right) and action potentials increased accordingly. Two of five neurons inactivated at the highest temperatures. 
( $\mathrm{pH}$ was adjusted to 7.4 with Tris-OH). A fine vibrated pipette dispersed neurons from the same region as that recorded in slices (Jin et al., 2004). All experiments began at room temperature $\left(\sim 22-23^{\circ} \mathrm{C}\right)$. Neurons were continuously superfused with a Y-tube system with complete exchanges within $20 \mathrm{~ms}$ (Murase et al., 1989). The in-line heating element controlled bath temperature, which was detected at the cell's location with a miniature temperature probe (TH-10Kmp; Cell MicroControls). Testing suggested that this probe likely underestimated temperature shifts experienced by cells. The probe sensing dimensions $(0.45 \times 2$ $\mathrm{mm}$ ) were much larger than neurons and temperature dropped steeply lateral to the flow center. Recordings were performed using MultiClamp 700B, pClamp, and electrodes filled with the following (in mM): $70 \mathrm{CsMeSO}_{4} 70$ $\mathrm{CsCl}, 5$ tetraethylammonium chloride, 10 EGTA, $2 \mathrm{NaATP}$, 0.3 NaGTP; and $\mathrm{pH}$ adjusted to 7.2 with Tris-OH. Neurons were voltage clamped to $-60 \mathrm{mV}$ and currents sampled at $20 \mathrm{kHz}$.

Data analysis. pClamp 9 and Mini Analysis (Synaptosoft) analyzed data off-line. Synaptic waveform values (decay-time constant and amplitude) and baseline values across each group were compared statistically using a minimum of $1 \mathrm{~min}$ at each condition using the Friedman repeated-measures ANOVA on ranks (frequency, decay time constant, and amplitude) or repeated-measures ANOVA (baseline, amplitude, and latency) with post hoc pairwise multiple comparisons (Holm-Sidak method, SigmaStat). All data are represented as mean \pm SEM and $p<$ 0.05 was considered significant.

Drugs. Capsaicin, NBQX, AP-5, TTX (1 $\mu \mathrm{M})$, and SB366791 were obtained from Tocris Bioscience and gabazine (SR-95531, GABA antagonist) from Sigma-RBI. Except where specifically noted otherwise, all recordings were made in $10 \mu \mathrm{M}$ gabazine to isolate glutamatergic EPSCs.

\section{Results}

Glutamate vesicles released from ST afferent terminals produce a background of spontaneous EPSC (sEPSC) activity that was higher in certain second-order NTS neurons under equivalent conditions $\left(33^{\circ} \mathrm{C}\right)$ (Fig. $\left.1 A\right)$. Neurons contacted by ST afferents that were activated by $100 \mathrm{nM}$ capsaicin (TRPV1+) had higher rates of sEPSCs than TRPV1 - neurons (Peters et al., 2010). Compared with controls at $33^{\circ} \mathrm{C}$, sEPSC activity decreased in TRPV1+ neurons when bath temperature was decreased and increased when temperature was raised. Thus, the rate of spontaneous EPSCs closely followed changes in bath temperature only in TRPV1+ NTS neurons (Fig. $1 B$ ), with the greatest changes nearest a physiological $37^{\circ} \mathrm{C}$. In contrast, in TRPV1- NTS neurons, the sEPSC rate was typically low at $33-34^{\circ} \mathrm{C}$ and changed little with temperature changes. Temperature-induced changes were rapid in frequency, showed no signs of diminishing over time, and were fully reversible. Event amplitudes and decay time constants (postsynaptic measures) changed similarly with weak associa-

B

C mIPSCs
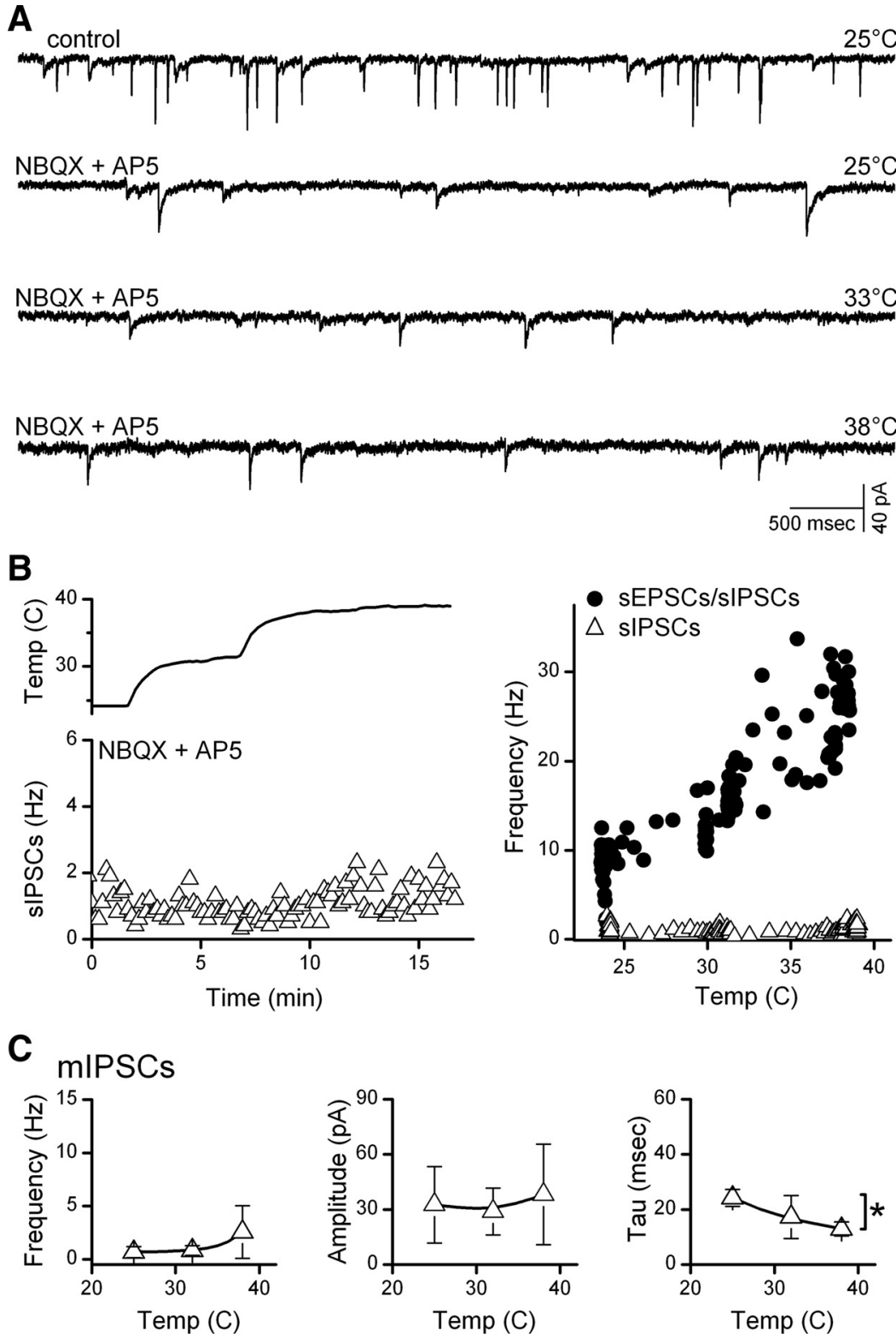

Figure 2. Temperature did not alter GABA release at second-order NTS neurons. A, Application of NBQX and AP-5 at $25^{\circ} \mathrm{C}$ greatly reduced total spontaneous synaptic activity (sEPSCs + sIPSCs; upper trace) and increases in temperature failed to substantially alter the remaining sIPSCs (lower three traces). $\boldsymbol{B}$, Counts of spontaneous IPSCS (triangles) changed little with temperature. Right, Temperature-evoked increases in total synaptic events (mEPSCs + mIPSCs; circles) were prevented by glutamate antagonists, indicating that in this neuron, sIPSCS were temperature insensitive (triangles). Note that gabazine eliminated the remaining events (data not shown) and these IPSCs were inwardly directed in our conditions. $C$, On average across TRPV $1+$ second-order neurons $(n=4)$, temperature produced no significant changes in mIPSC (triangles) frequency $(p=0.18)$ or amplitude $(p=$ 0.27 ), and small decreases in time constant (tau, $p=0.01$ ). Asterisk marks significant differences.

tions with TRPV1 subtype, suggesting that presynaptic properties of TRPV1+ terminals were the most temperature-sensitive (Fig. 1C). Consistent with this idea, the TRPV1-selective antagonist SB366791 attenuated temperature-induced increases in mEPSC rate in TRPV1+ neurons $(n=5, p<0.001$, two-way repeated-measures ANOVA) (Fig. 1D).

The inward flux of cations through open TRPV1 channels should promote glutamate release by depolarizing ST synaptic terminals, triggering voltage-activated processes and/or calcium permeation via TRPV1. To test whether voltage-activated $\mathrm{Na}^{+}$ channels contributed to basal release, we applied $1 \mu \mathrm{M}$ TTX and measured spontaneously occurring miniature EPSCs (mEPSCs) 
A

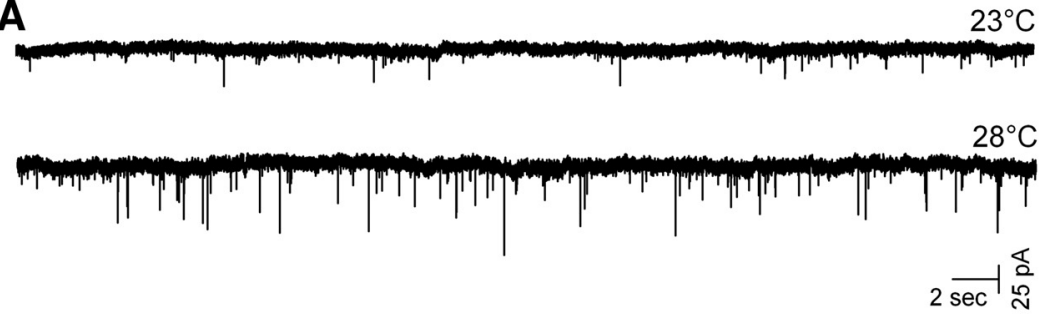

B

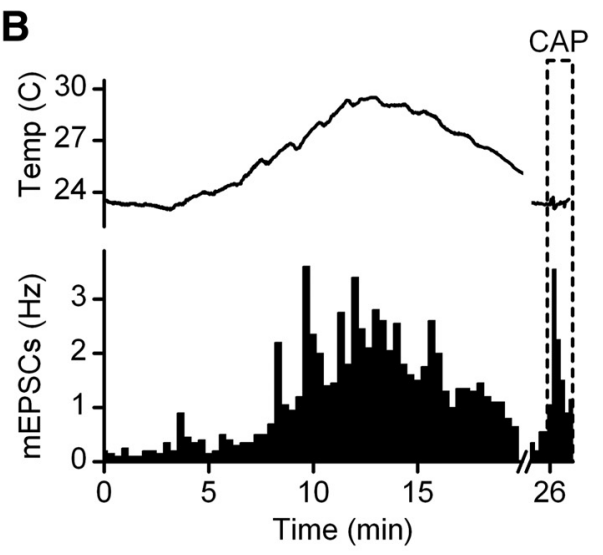

C
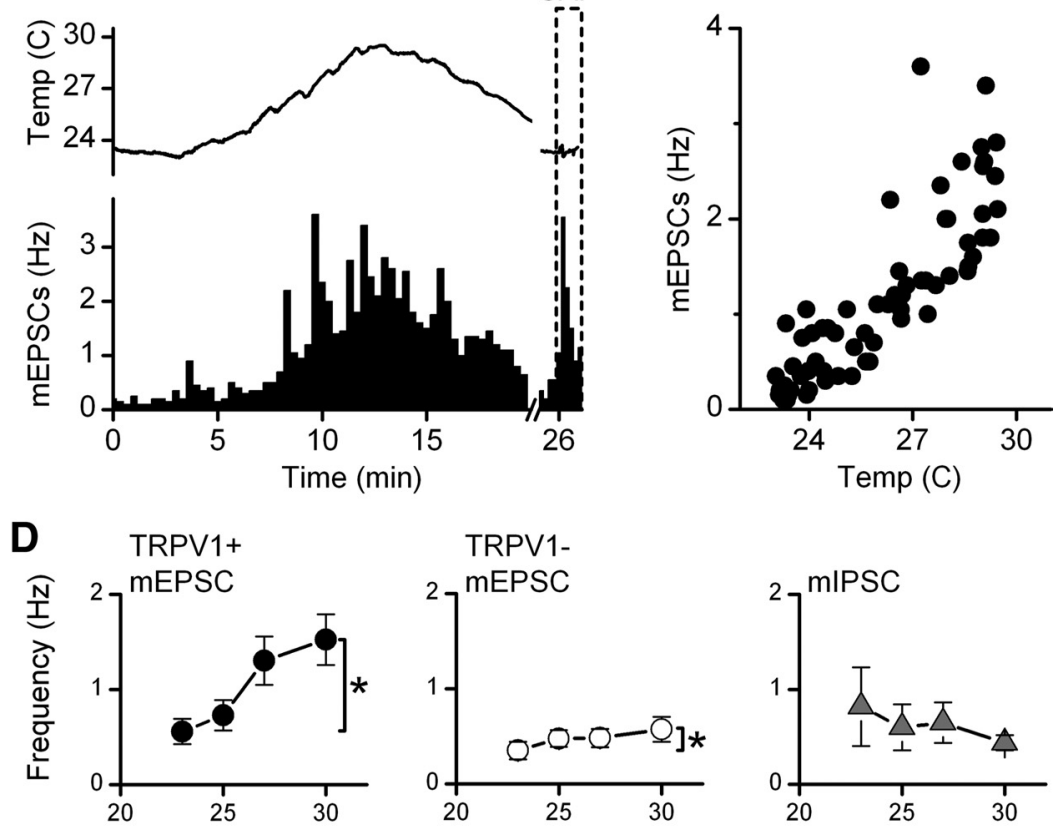

TRPV1-
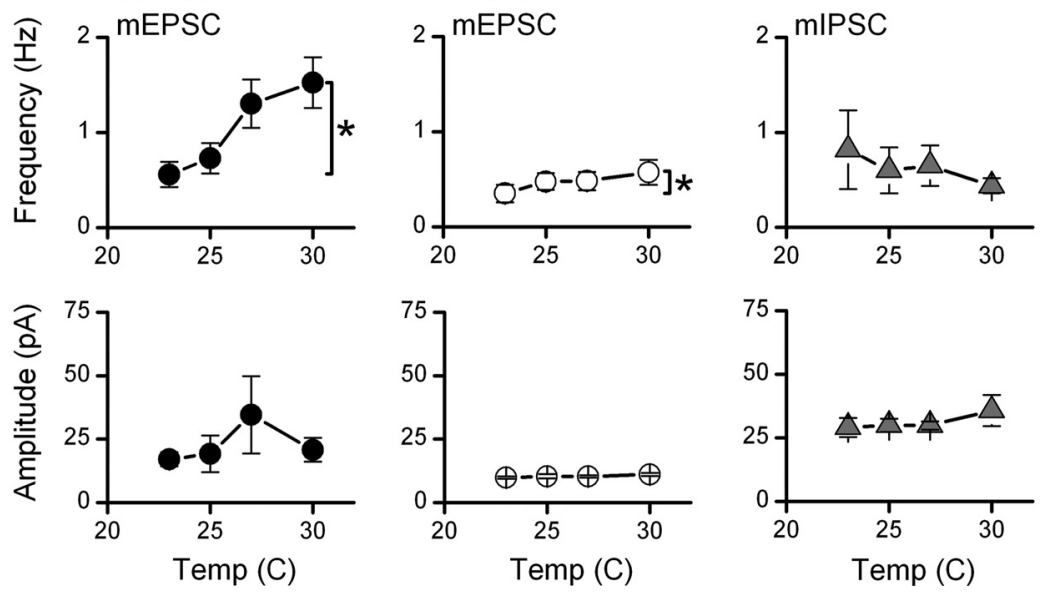

Figure 3. Temperature-sensitive TRPV1+ synaptic responses were preserved in isolated NTS neurons. $A$, Rates of mEPSC activity $\left(1 \mu \mathrm{M}\right.$ TTX) in mechanically dispersed NTS neurons at room temperature $\left(23^{\circ} \mathrm{C}\right)$ increased substantially with temperature. A probe placed close to the position of the isolated cell registered a rise and then fall in temperature that evoked an increase and decrease of $m E P S C$ rate in this representative TRPV1 + neuron. $\boldsymbol{B}$, Temperature increases reversibly increased mEPSCs (black bars), as did capsaicin (CAP; $100 \mathrm{~nm}$, dashed-border bar). $C$, The average $\mathrm{mEPSC}$ rate $(20 \mathrm{~s} \mathrm{bin)}$ increased linearly with temperature in this range for this neuron. Gabazine $(10 \mu \mathrm{M})$ was present throughout. $\boldsymbol{A}-\boldsymbol{C}$ were taken from same neuron. Note that the large probe likely underestimated actual temperature at neuron. $\boldsymbol{D}$, In aggregate average, only mEPSC frequency for TRPV1+ $(n=10$ neurons; filled circles) was steeply temperature-sensitive, whereas TRPV1 - mEPSCs ( $n=5$ neurons, unfilled circles) and mIPSCS ( $n=8$ neurons, triangles) from both TRPV1 + and TRPV1 - types were insensitive. Amplitudes were uniformly unaltered. Group data are means \pm SEM. ${ }^{*} p<0.02$. EPSCs isolated using gabazine $(10 \mu \mathrm{M})$ and IPSCs isolated in NBQX and AP-5.

(Fig. $1 D$ ). Similar to sEPSCs, changes in temperature produced new steady levels of mEPSC activity that persisted at each temperature in TRPV1+ neurons. Although the mean sEPSC and mEPSC results are based on separate studies of two different groups of TRPV1+ neurons, the mEPSC average temperature relationship was quantitatively similar to that for sEPSCs. Like the sEPSCs, mEPSC amplitudes increased only slightly and decay time constants decreased with temperature ( $p<0.001$, data not shown). Reduction of external calcium from 2 to $1 \mathrm{~mm}$ decreased the $\mathrm{mEPSC}$ rate and the response to temperature (Fig. 1D). Under current clamp, synaptic potential activity increased steeply with tem- perature in all cells and increased action potential activity in most (three of five neurons) (Fig. 1E). Together, our results suggest that temperature acts on TRPV1 at ST afferents to promote vesicular glutamate release independent of action potential activity.

Second-order NTS neurons generally receive both excitatory and inhibitory synaptic contacts. To test whether IPSCs were temperature-sensitive, we recorded miniature synaptic events in TRPV1+ neurons (Fig. 2A). Counts of total synaptic activity-mEPSCs and miniature IPSCs (mIPSCs) - increased in direct proportion to temperature (Fig. $2 B$, right). However, blockade of glutamate receptors with NBQX and AP-5 reduced synaptic activity to a low level of remaining IPSCs that were insensitive to temperature changes (Fig. $2 A, B)$. On average $(n=4)$, mIPSC frequency, amplitudes, and kinetics were minimally altered within the range of $25^{\circ} \mathrm{C}$ to $38^{\circ} \mathrm{C}$ (Fig. $2 \mathrm{C}$ ). Together, these results in slices indicate that substantial temperature sensitivity in NTS required TRPV1-a factor only expressed presynaptically within one type of ST afferent terminal but not present postsynaptically or in presynaptic GABAergic terminals.

To better understand the mechanisms of action of TRPV1 and reduce the possibility of interactions with adjacent neurons, we mechanically isolated medial NTS neurons using a microharvesting approach that retained functioning synaptic terminals (Jin et al., 2010). All neurons displayed glutamatergic and GABAergic events. Raising temperature of the perfusion solution increased mEPSC activity substantially in many isolated neurons (Fig. $3 A, B$ ). The timing of temperature changes was estimated using a probe placed close to the recorded neuron. The frequency of mEPSC activity closely followed changes in temperature (Fig. $3 B, C$ ). The values for temperature registered at the cell location were consistently $\sim 5^{\circ} \mathrm{C}$ lower in single cell experiments than in slices and we likely underestimated the actual temperatures at the cells. Outflow temperatures of $35^{\circ} \mathrm{C}$ reached $29-30^{\circ} \mathrm{C}$ near the cell location but moving the probe closer to the outlet did not reduce the difference and we conclude that, most likely, the probe is too large to accurately register the temperature. Note that all neurons with highly temperature-dependent mEPSCs also responded to capsaicin with increases in mEPSC frequency (Fig. 3B, lower right). Temperature-insensitive neurons were capsaicin-insensitive (results not shown). On average across neurons (Fig. 3D), temperature selectively increased mEPSC frequency of TRPV $1+$ neurons $(n=10)$ but not in TRPV1 - neurons $(n=5)$. The mIPSC frequency was not changed by temperature whether recorded in TRPV $1+$ or TRPV1 - neurons (Fig. 3D). The amplitudes of mEPSCs or 

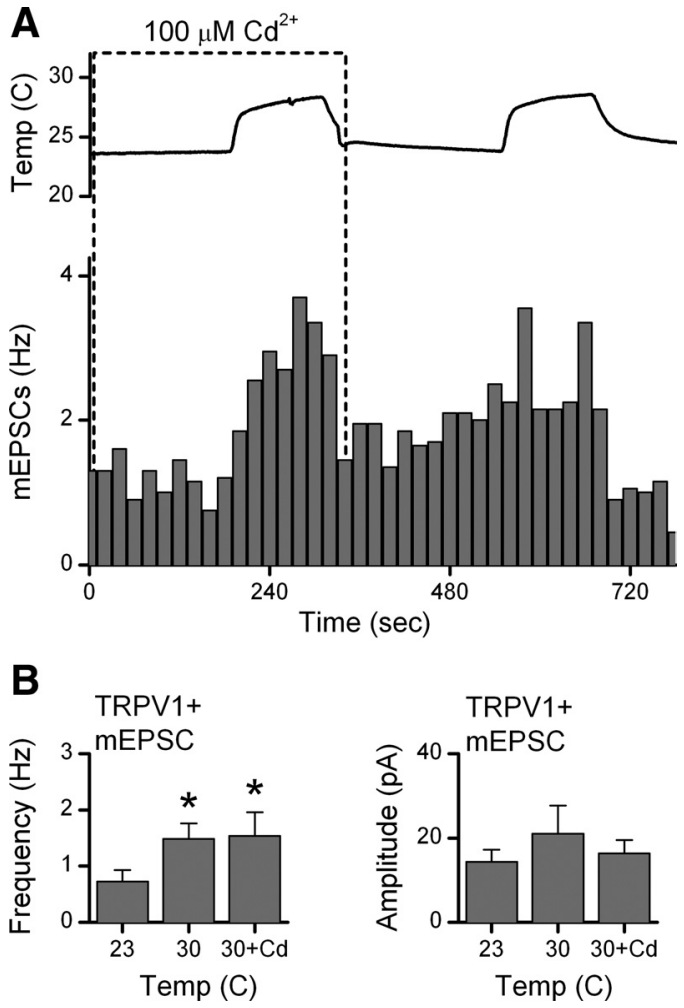

Figure 4. Temperature-activated TRPV1 release of glutamate is calcium-dependent. Raising temperature substantially increased mEPSC frequency in this representative isolated TRPV1+ neuron $(\boldsymbol{A}, p<0.01)$, even in the presence of the nonselective calcium channel antagonist $\mathrm{Cd}^{2+}\left(100 \mu \mathrm{M}\right.$, dashed-border box). On average ( $\boldsymbol{B}, n=7$ neurons), $\mathrm{Cd}^{2+}$ did not change the evoked increase in $\mathrm{mEPSC}$ rate to raising temperature from 23 to $30^{\circ} \mathrm{C}(p>0.7)$. Amplitudes were unaltered by $\mathrm{Cd}^{2+}(p>0.2)$. Gabazine $(10 \mu \mathrm{m})$ present throughout. Capsaicin response not shown. ${ }^{*} p<0.02$, from control.

mIPSCs in isolated neurons were insensitive to temperature (Fig. 3D).

TRPV1 channels are more permeable to $\mathrm{Ca}^{+2}$ than to $\mathrm{Na}^{+}$ (Caterina et al., 1997; Chung et al., 2008). Capsaicin activation of TRPV1 triggered glutamate release onto NTS neurons independent of voltage-activated $\mathrm{Ca}^{+2}$ channels (VACC) (Jin et al., 2004). To test whether VACC contributed to temperatureactivated glutamate release, we measured mEPSC increases to temperature step increases in isolated NTS neurons and added $\mathrm{Cd}^{2+}(100 \mu \mathrm{M})$ to block all VACCs nonselectively. Rising temperatures evoked rapid increases in mEPSCs frequency (Fig. 4), even in the presence of $\mathrm{Cd}^{2+}$. Temperature-evoked increases in mEPSC rates, on average $(n=7)$ (Fig. $4 B)$, more than doubled $(p<0.02)$, and these increases were similar with and without $\mathrm{Cd}^{2+}$ present $(p=0.80)$. Amplitudes of mEPSCs were unaltered by temperature or $\mathrm{Cd}^{2+}(p>0.1)$. Together, our results suggest that temperature activates TRPV 1 channels, which allow calcium entry into the terminals to increase the rate of glutamate release and this process does not require VACCs.

\section{Discussion}

The present results revealed profound differences in the regulation of autonomous release of glutamate vesicles between two classes of afferent terminals at second-order NTS neurons. Our new findings include the following: (1) physiological temperatures $\left(\sim 36-38^{\circ} \mathrm{C}\right)$ drive high rates of spontaneous glutamate release only from TRPV1+ afferent terminals; (2) TRPV1- and GABAergic terminals are nearly temperature insensitive; (3) tem- perature responses involved calcium-dependent, tonic glutamate release without a requirement for voltage-gated $\mathrm{Na}^{+}$or $\mathrm{Ca}^{2+}$ channel activity; and (4) TRPV1-specific antagonism attenuated thermally evoked glutamate release, suggesting that TRPV1 controls this pool of vesicles.

TRPV1 receptors on presynaptic ST terminals confer prominent temperature sensitivity to spontaneous glutamate release. The steep sensitivity of sEPSC and mEPSC regulation between 30 and $40^{\circ} \mathrm{C}$ only in TRPV $1+$ terminals contrasts with similar evoked synchronous release at these same ST terminals (Peters et al., 2010). In fact, regulation of the synchronous vesicle pool is indistinguishable in most respects between TRPV1+ and TRPV1 - ST terminals (Andresen and Peters, 2008; Peters et al., 2010). The temperature sensitivity of mEPSC rates from TRPV1+ terminals on NTS neurons greatly exceeds that of AMPA-mediated EPSCs in other central neurons (Hestrin et al., 1990) and the peripheral discharge from cranial afferents, including baroreceptors, is much less temperature-sensitive (McQueen and Eyzaguirre, 1974). At physiological temperatures, the absolute sEPSC rate at TRPV1 + ST neurons averaged $\sim 25$ mEPSCs per second-a rate 20-30-fold greater than TRPV1- neurons, which had a rate typical of most central synapses (Wasser and Kavalali, 2009). This high rate of release was diagnostic of TRPV1 + terminals in NTS. Single ST afferent axons contribute $\sim 20$ somatodendritic synaptic contacts, whether from TRPV1+ or TRPV1 - afferents (Andresen and Peters, 2008). The attenuation of autonomous release to low levels during cooling and TRPV1 block suggests that most sEPSCs arise from ST afferents. Likely other glutamate terminals, including from NTS interneurons, contributed to our total activities, but evidence suggests that their numbers are relatively low compared with ST contacts (McDougall et al., 2009). FM1-43 tracking of multiple individual terminals on NTS neurons shows that capsaicin and potassium depolarization activated the same terminals (Jin et al., 2010), a finding consistent with colocalization of the TRPV1-dependent pool with the depolarization-evoked vesicle pool.

TRPV1 contains a nonselective cation pore that is gated by heat (typically $>40^{\circ} \mathrm{C}$ ), protons $(\mathrm{pH}, \sim 5)$, and/or vanilloid chemicals to transduce potentially harmful conditions (Lumpkin and Caterina, 2007)-conditions unlikely to exist within the normal CNS. Interestingly, TRPV1 blockers are multimodal with different potencies for thermal versus vanilloid gating (Garami et al., 2010) and this may explain why there is only partial block of release by SB-366791 (Peters et al., 2010). TRPV1 thermosensitivity likely arises from a unique portion of the pore that functions independently of the capsaicin binding/gating motif (Yang et al., 2010). We found previously that low temperature was always more effective than pharmacological blockade (Peters et al., 2010). This is consistent with a relative resistance of TRPV1 thermal responses to many high affinity vanilloid blockers (Garami et al., 2010), and the more complete block at low temperatures suggests that most glutamate terminals are likely afferent in origin. Our results suggest important distinctions among evoked, asynchronous, and spontaneous vesicle release but with certain common elements in their control. All glutamate release modes show similar dependence on external $\mathrm{Ca}^{2+}$ entry (Peters et al., 2010). However, VACCs similarly contribute to synchronous and asynchronous release but are not required for spontaneous release. Spontaneous synaptic events are considered to be random vesicle fusions that closely correlate with evoked events (Hestrin, 1992). Our present results show that activity-independent release from ST terminals is actively controlled by TRPV1. Spontaneous synaptic signals influence dendritic protein synthesis patterns and 
postsynaptic receptivity to subsequent evoked events (Sutton et al., 2004). The much higher rates of TRPV1+ sEPSCs and their regulation by peptides (Appleyard et al., 2005; Peters et al., 2008) may represent a robust ST afferent signal that helps to phenotypically differentiate NTS neurons (Bailey et al., 2002).

The physiological consequences of spontaneous glutamate signaling in NTS are not clear, but contrasts between A- and $\mathrm{C}$-fiber afferents are apparent. First, this tonic glutamate signal arises from C-type afferents that are often physiologically silent under normal circumstances. Second, unmyelinated cranial visceral afferents represent up to $90 \%$ of all afferents arising from different organs (Andresen et al., 1978; Jammes et al., 1982; Kubin and Davies, 1995). From one typical visceral afferent source common to medial NTS, $75 \%$ of aortic baroreceptors were unmyelinated and inactive at normal blood pressures (Andresen and Kunze, 1994). Thus, C-fiber baroreceptors represent physiologically silent afferents (Jones and Thorén, 1977); a finding similar for other organs (Coleridge and Coleridge, 1984). Since activity-dependent processes importantly shape and maintain patent synaptic connections (McKinney et al., 1999; Poo, 2001; Sutton et al., 2004), TRPV1-dependent, activity-independent generation of glutamate transmission may maintain the afferent signaling from C-type axons.

\section{References}

Andresen MC, Kunze DL (1994) Nucleus tractus solitarius: gateway to neural circulatory control. Annu Rev Physiol 56:93-116.

Andresen MC, Peters JH (2008) Comparison of baroreceptive to other afferent synaptic transmission to the solitary tract nucleus. Am J Physiol Heart Circ Physiol 295:H2032-H2042.

Andresen MC, Krauhs JM, Brown AM (1978) Relationship of aortic wall baroreceptor properties during development in normotensive and spontaneously hypertensive rats. Circ Res 43:728-738.

Andresen MC, Doyle MW, Bailey TW, Jin YH (2004) Differentiation of autonomic reflex control begins with cellular mechanisms at the first synapse within the nucleus tractus solitarius. Braz J Med Biol Res 37:549-558.

Appleyard SM, Bailey TW, Doyle MW, Jin YH, Smart JL, Low MJ, Andresen MC (2005) Proopiomelanocortin neurons in nucleus tractus solitarius are activated by visceral afferents: regulation by cholecystokinin and opioids. J Neurosci 25:3578-3585.

Bailey TW, Jin YH, Doyle MW, Andresen MC (2002) Vanilloid sensitive afferents activate neurons with prominent A-type potassium currents in nucleus tractus solitarius. J Neurosci 22:8230-8237.

Caterina MJ, Schumacher MA, Tominaga M, Rosen TA, Levine JD, Julius D (1997) The capsaicin receptor: a heat-activated ion channel in the pain pathway. Nature 389:816-824.

Chung MK, Güler AD, Caterina MJ (2008) TRPV1 shows dynamic ionic selectivity during agonist stimulation. Nat Neurosci 11:555-564.

Coleridge JC, Coleridge HM (1984) Afferent vagal C fibre innervation of the lungs and airways and its functional significance. Rev Physiol Biochem Pharmacol 99:1-110.

Doyle MW, Andresen MC (2001) Reliability of monosynaptic transmission in brain stem neurons in vitro. J Neurophysiol 85:2213-2223.

Garami A, Shimansky YP, Pakai E, Oliveira DL, Gavva NR, Romanovsky AA
(2010) Contributions of different modes of TRPV1 activation to TRPV1 antagonist-induced hyperthermia. J Neurosci 30:1435-1440.

Hestrin S (1992) Developmental regulation of NMDA receptor-mediated synaptic currents at a central synapse. Nature 357:686-689.

Hestrin S, Sah P, Nicoll RA (1990) Mechanisms generating the time course of dual component excitatory synaptic currents recorded in hippocampal slices. Neuron 5:247-253.

Jammes Y, Fornaris E, Mei N, Barrat E (1982) Afferent and efferent components of the bronchial vagal branches in cats. J Auton Nerv Syst 5:165-176.

Jin YH, Bailey TW, Li BY, Schild JH, Andresen MC (2004) Purinergic and vanilloid receptor activation releases glutamate from separate cranial afferent terminals. J Neurosci 24:4709-4717.

Jin YH, Zhang Z, Mendelowitz D, Andresen MC (2009) Presynaptic actions of propofol enhance inhibitory synaptic transmission in isolated solitary tract nucleus neurons. Brain Res 1286:75-83.

Jin YH, Cahill EA, Fernandes LG, Wang X, Chen W, Smith SM, Andresen MC (2010) Optical tracking of phenotypically diverse individual synapses on solitary tract nucleus neurons. Brain Res 1312:54-66.

Jones J, Thorén PN (1977) Characteristics of aortic baroreceptors with nonmedullated afferents arising from the aortic arch of rabbits with chronic renovascular hypertension. Acta Physiol Scand 101:286-293.

Julius D, Basbaum AI (2001) Molecular mechanisms of nociception. Nature 413:203-210.

Kubin L and Davies RO (1995) Central pathways of pulmonary and airway vagal afferents. In: Regulation of Breathing (Dempsey JA, Pack AI, eds), pp. 219-284. Marcel Dekker, New York.

Lumpkin EA, Caterina MJ (2007) Mechanisms of sensory transduction in the skin. Nature 445:858-865.

McDougall SJ, Peters JH, Andresen MC (2009) Convergence of cranial visceral afferents within the solitary tract nucleus. J Neurosci 29:12886-12895.

McKinney RA, Capogna M, Dürr R, Gähwiler BH, Thompson SM (1999) Miniature synaptic events maintain dendritic spines via AMPA receptor activation. Nat Neurosci 2:44-49.

McQueen DS, Eyzaguirre C (1974) Effects of temperature on carotid chemoreceptor and baroreceptor activity. J Neurophysiol 37:1287-1296.

Murase K, Ryu PD, Randic M (1989) Excitatory and inhibitory amino acids and peptide-induced responses in acutely isolated rat spinal dorsal horn neurons. Neurosci Lett 103:56-63.

Patterson LM, Zheng H, Ward SM, Berthoud HR (2003) Vanilloid receptor (VR1) expression in vagal afferent neurons innervating the gastrointestinal tract. Cell Tissue Res 311:277-287.

Peters JH, McDougall SJ, Kellett DO, Jordan D, Llewellyn-Smith IJ, Andresen MC (2008) Oxytocin enhances cranial visceral afferent synaptic transmission to the solitary tract nucleus. J Neurosci 28:11731-11740.

Peters JH, McDougall SJ, Fawley JA, Smith SM, Andresen MC (2010) Primary afferent activation of thermosensitive TRPV1 triggers asynchronous glutamate release at central neurons. Neuron 65:657-669.

Poo MM (2001) Neurotrophins as synaptic modulators. Nat Rev Neurosci $2: 24-32$.

Sun H, Li DP, Chen SR, Hittelman WN, Pan HL (2009) Sensing of blood pressure increase by transient receptor potential vanilloid 1 receptors on baroreceptors. J Pharmacol Exp Ther 331:851-859.

Sutton MA, Wall NR, Aakalu GN, Schuman EM (2004) Regulation of dendritic protein synthesis by miniature synaptic events. Science 304:1979-1983.

Wasser CR, Kavalali ET (2009) Leaky synapses: regulation of spontaneous neurotransmission in central synapses. Neuroscience 158:177-188.

Yang F, Cui Y, Wang K, Zheng J (2010) Thermosensitive TRP channel pore turret is part of the temperature activation pathway. Proc Natl Acad Sci U S A 107:7083-7088. 